\author{
صفورا شهروان' و تقى قورجى
}

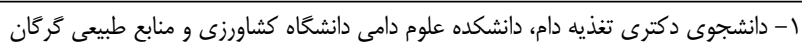

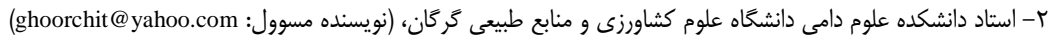

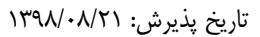 \\ IV صفحه: 9 تا

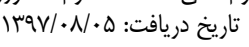

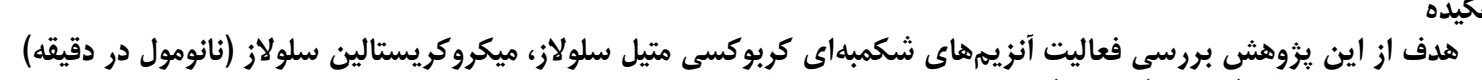

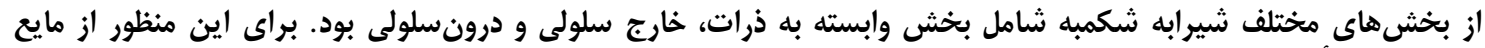

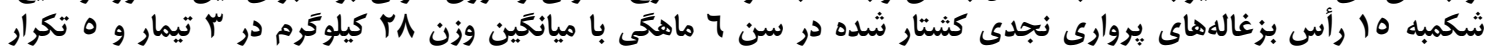

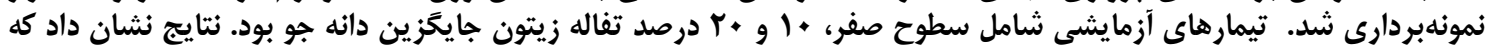

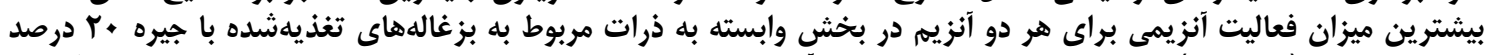

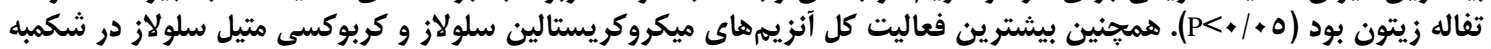

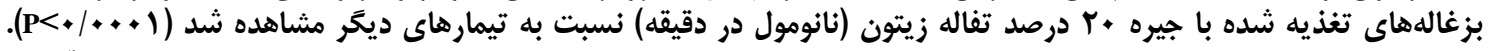

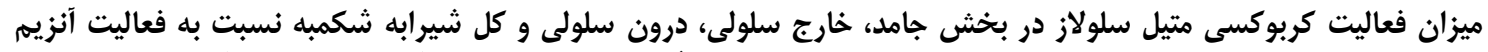

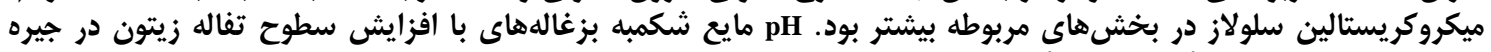

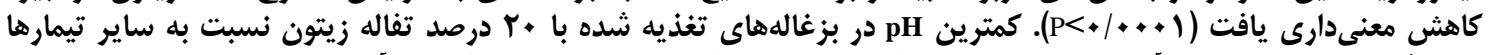

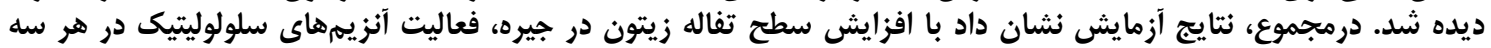

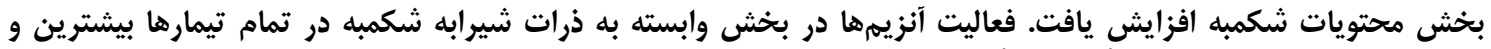

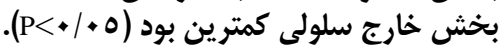

وازههاى كليدى: كربوكسى متيل سلولاز، ميكروكريستالين سلولاز، آنزيه، بزغالههاى كثتار شده، تفاله زيتون

ميكروكر يستالين سلولاز به قسمت انتهايى آزاد زنجيره حمله

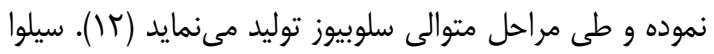

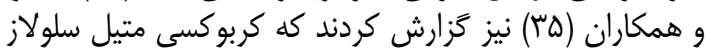

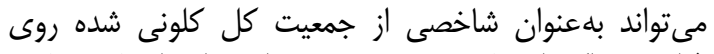

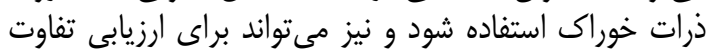

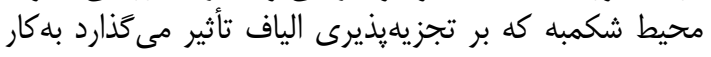

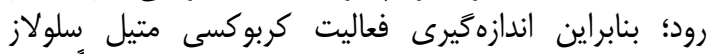

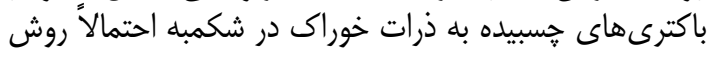

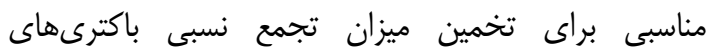

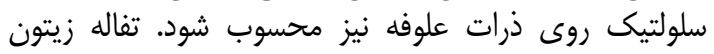

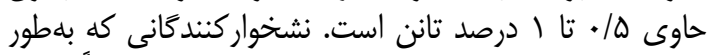

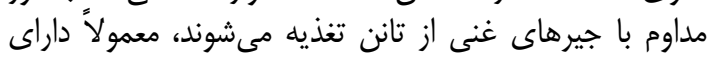

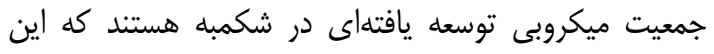

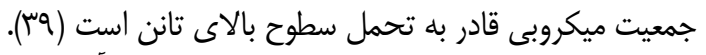

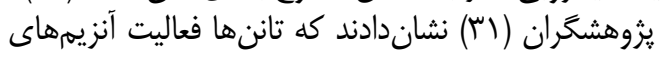

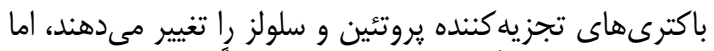

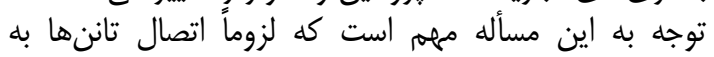

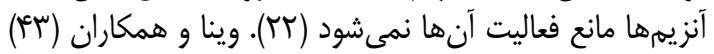

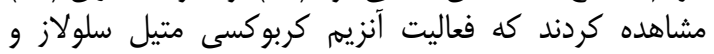

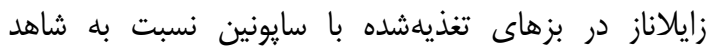

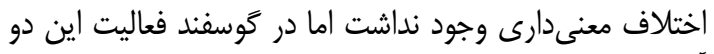

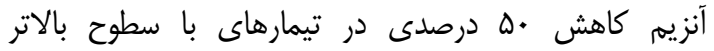

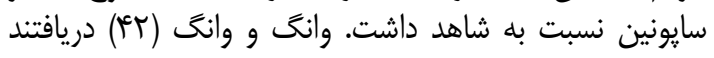

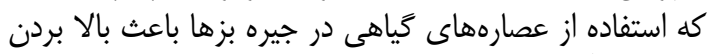

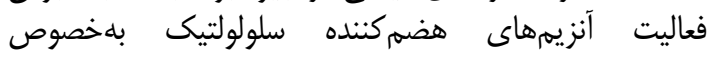
ميكروكريستالين و كربو كسى متيل سلولاز شد. با تحقيقى كه به

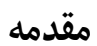

قسمت عمده خوراك نشخواركنندكان را ديواره سلول

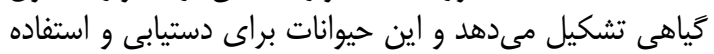

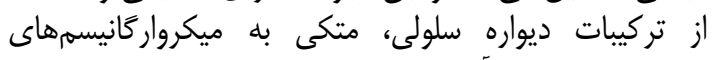

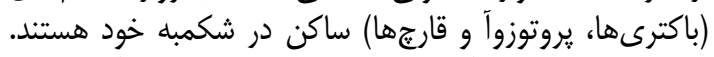

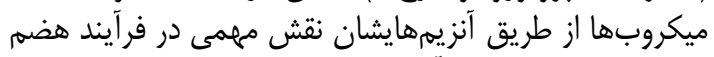

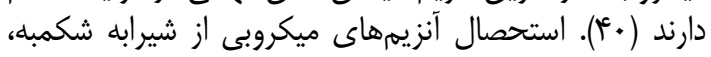

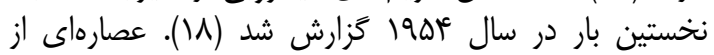

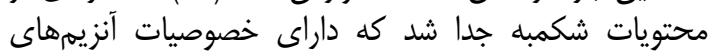

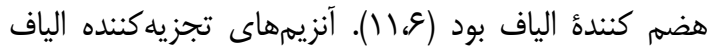

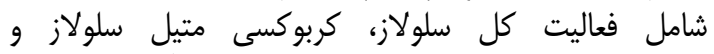

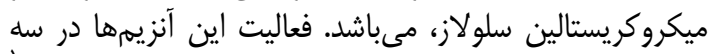

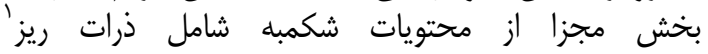

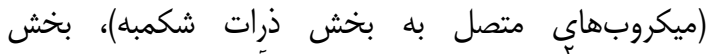

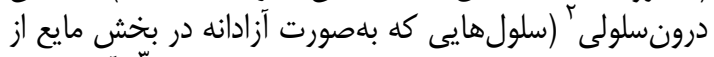

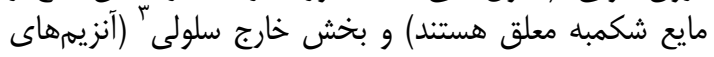

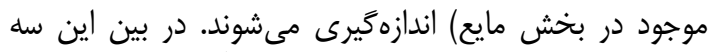

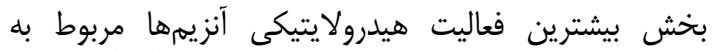

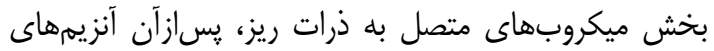

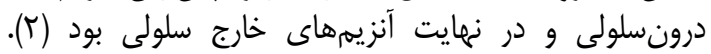

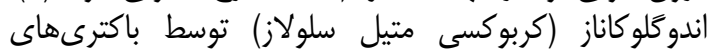

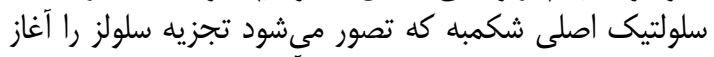

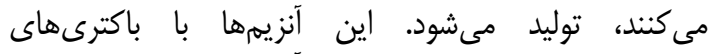

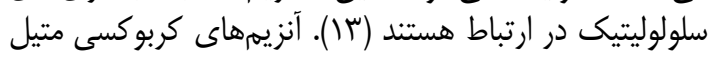

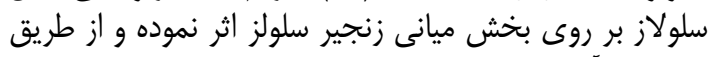
هيدروليز آنرا قطع كرده و توليد دوزنجيره كوتاهتر مى كند. اما 
درون سلولى (باكترىها، قارجها و بروتوزواً)، خارج سلولى إنى

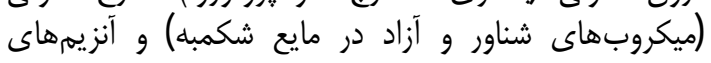

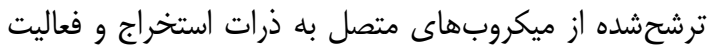

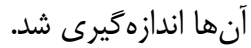
جمع آورى مايع شكمبرى شنديه

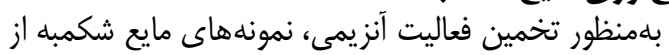

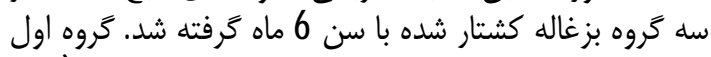

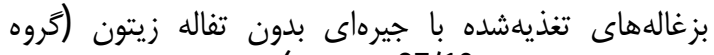

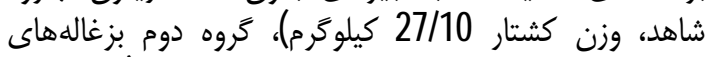

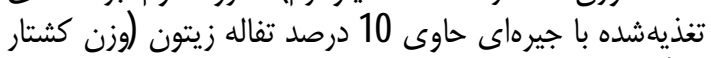

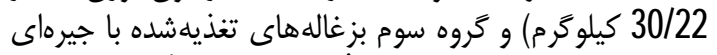

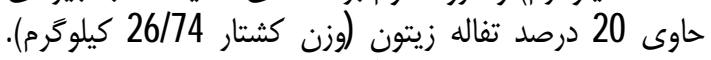

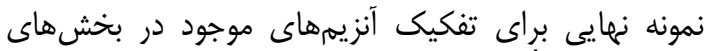

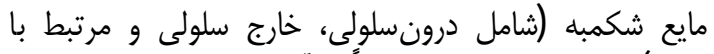
ذرات) در يك فلاكس كرم فوراً به آزمايشكاه منتقل شدار

استخراج آنز يمها آناكيا

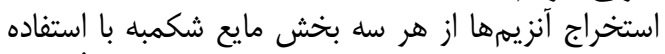

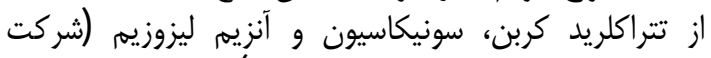

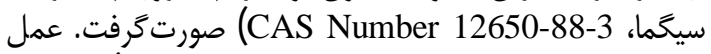

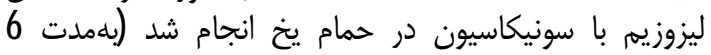

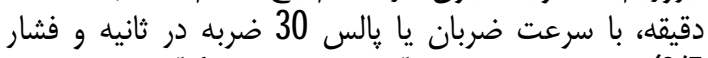

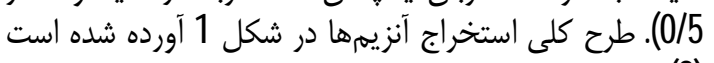

وانگ و همكاران (41) بر روى بزهاى در حال رشد انجام

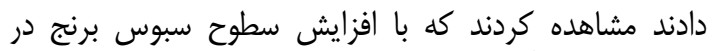

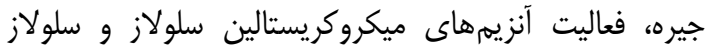

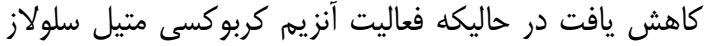

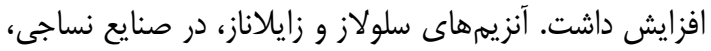

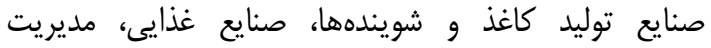

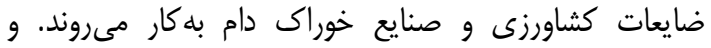

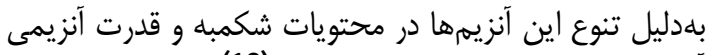

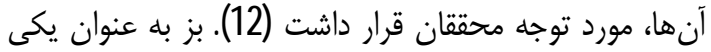

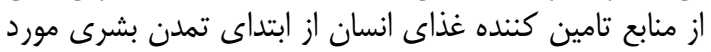

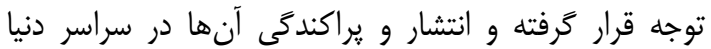
ديده مى شود (17، (17) هدف از اين مطالعه بررسى تأثير سطوح مختلف تفاله

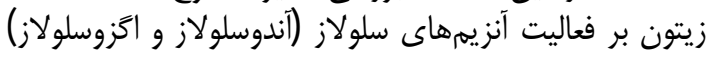

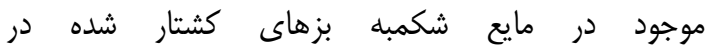
كشتاركاه مىباشد. در اين زمينه تحقيقات خيلى كمى صنى صورت

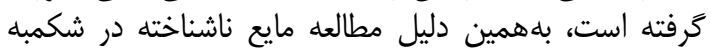
بزغالههاى كشتار شده ضرورى به نظر مى رسلد.

مواد و روشها روش اصلى درو اين آزمايش روند شرح دادهشده توسط إسار

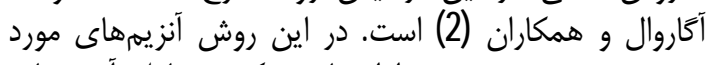
بررسى در سه بخش مجزا ان از مايع شكمبه شامل آنزيمهاى

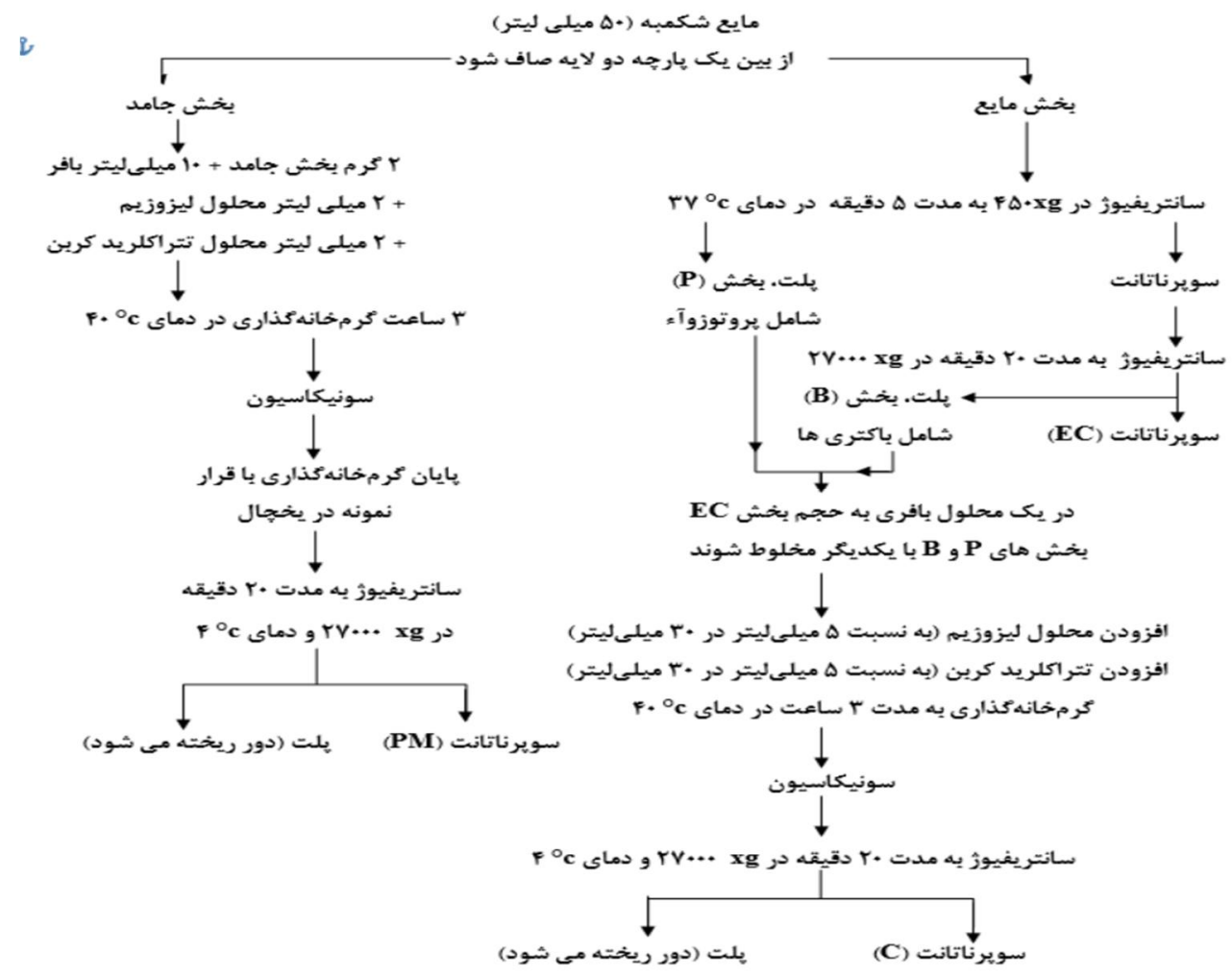

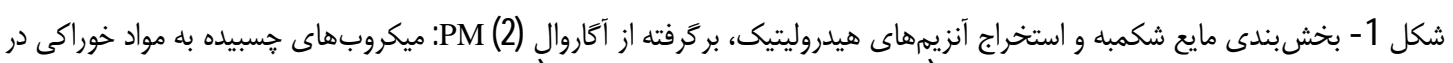

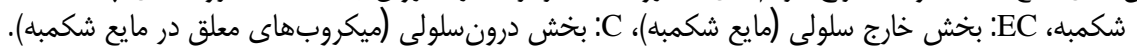

Figure 1. Fractionation of rumen contents and extraction of hydrolytic enzymes, Agarwal (2) PM:Particulate Material, EC: Extracellular, C: Cellul 
اختلاف جذبات نورى نمونههاى مورد آزمايش از شاهد محاسبه

محاسبات

$$
\text { تغييرات A }
$$

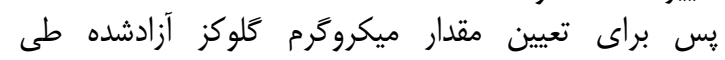

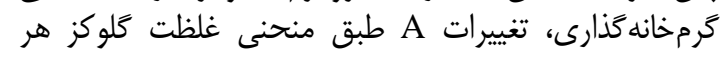

نمونه تعيين مى شودان

لمونه تعيين مى شانوخ: كلوكز /ميلى ليتر/دقيقه)

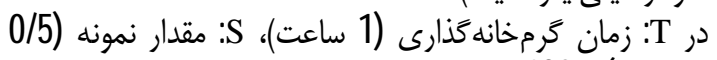
ميلى ليتر) و1800: وزن موان مولكولى كلوكز

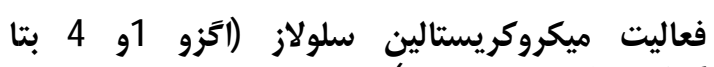

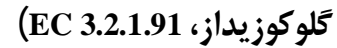
ميكروكريستالين سلولز همان آويسل است. روش محاسبه آنزيمهاى تجزيهكننده آويسل بهانز آنزيم كربوكسى متيل سلولاز است: 1) آويسل 1 آن درصد

2) مخلوط مورد آزمايش: شامل 1 ميلىليتر محلول آويسل و

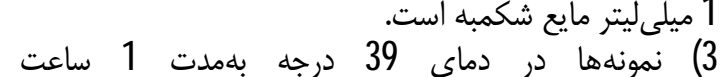
كرمخانه گذارى شدناند

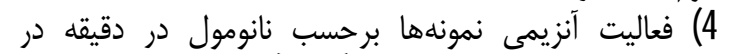

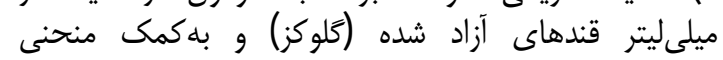

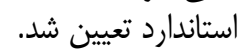
براى استاندارد سازى كلوكز از معادله زير استفاده شد. $\mathrm{Y}=2.6287 \mathrm{X}-0.009$

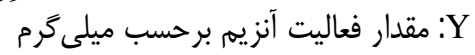

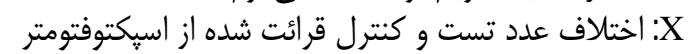
$\mathrm{R}^{2}=0.9917$

اين آزمايش در قالب طرح كاملا تصادفى و با استفاده از

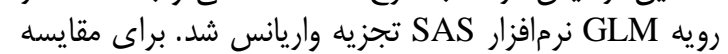

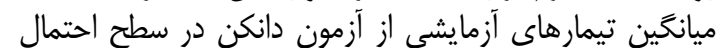
95 درصد استفاده شد.
فعاليت كربوكسى متيل سلولاز (اندو 1و 4 بتا (EC 3.1.4 ملوكوزيدازيت

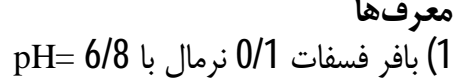

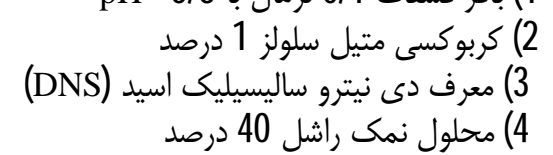

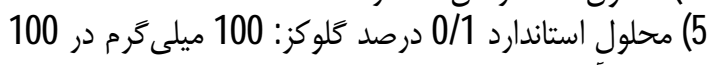

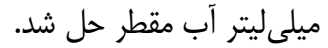
روش كار 1) لوله (a) شاهد: 1 ميلى ليتر بافر فسفات،015 ميلى ليتر نمونه

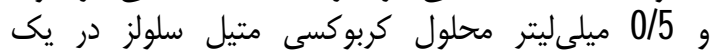

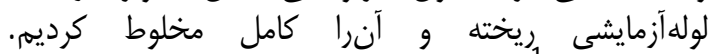

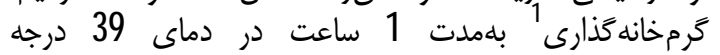
سانتى 2 واكنش متوقف شد.

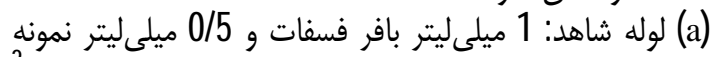

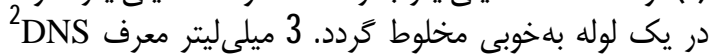

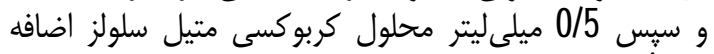

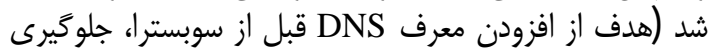
از واكنش آنزيمهاى مايع شكمبه با سوبسترا است).

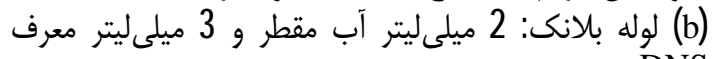
DNS

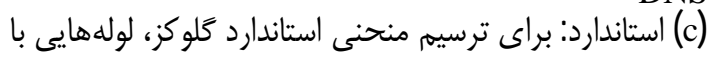

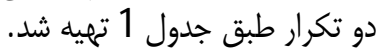

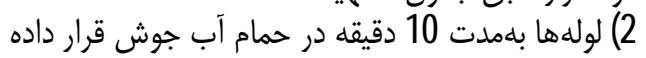
شدنلد 3) بههر لوله 1 ميلىليتر محلول نمك راشل افزوده شد و لولهها در دماى اتاق خنى لولى شدند

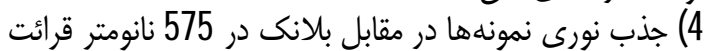

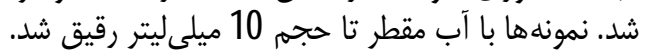
5) منحنى ويمايش (كاليبراسيون) استانداردهاى كلى ملوكز

Table 1. The standard method for glucose measurement

\begin{tabular}{|c|c|c|c|c|c|c|c|c|c|}
\hline$\underline{c}$ & $\varepsilon$ & 7 & $E$ & 5 & 4 & $\Xi$ & 2 & ] & شماره لوله \\
\hline C & $C / 2$ & C/5C & $C / T$ & ] & $1 / 2$ & $1 / 5 \mathrm{C}$ & $1 / 75$ & 2 & آب مقطر (ml) \\
\hline 2 & $1 / \pi$ & $1 / 5 C$ & $1 / 2 E$ & ] & $C / \pi$ & $C / 5 C$ & $C / 2 E$ & $\mathrm{C}$ & محلول استاندارد كلوكز (ml) \\
\hline 2000 & $175 C$ & $150 \mathrm{C}$ & 1250 & 1000 & $75 C$ & 500 & EC & $\mathrm{C}$ & غلظت كلوكز ( \\
\hline$\Xi$ & $\Xi$ & $\Xi$ & $\Xi$ & $\Xi$ & $\Xi$ & $\Xi$ & $\Xi$ & $\Xi$ & معرف (ml) DNS \\
\hline
\end{tabular}

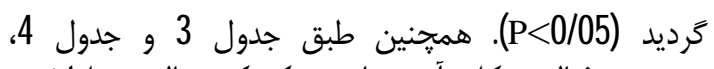

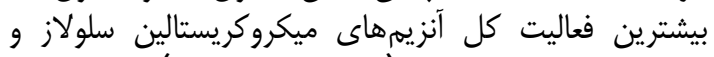

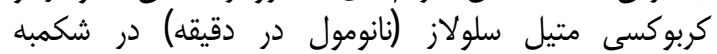

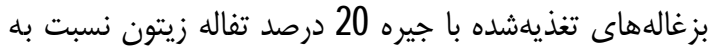

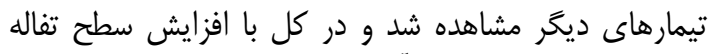

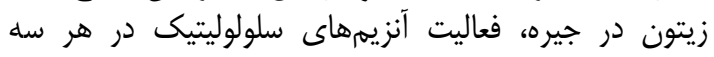

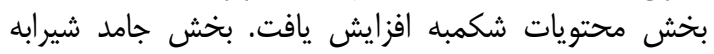

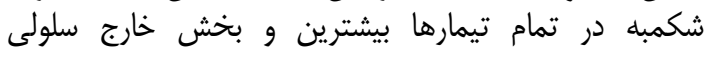

جدول1 - نحوه استانداردسازى براى قرائت كلوكز

\section{نتايج و بحث \\ فعاليت آنزيمهاى سلو سلاز}

فعاليت آنزيمهاى سلولوليماليتيك (كربوكسى متيل سلولاز،

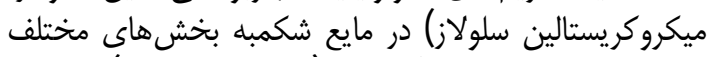

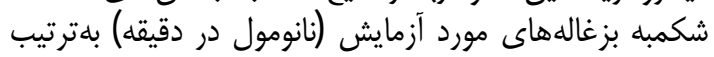

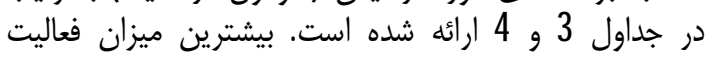

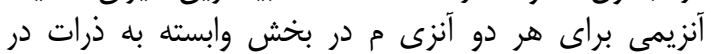

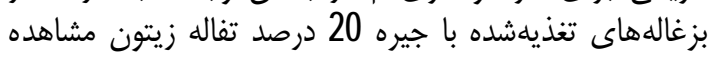


و 838/89، 246/14، 239/20, 351/55 (نانومول در دقيقه) بود

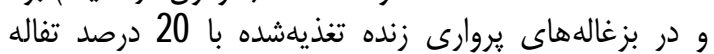

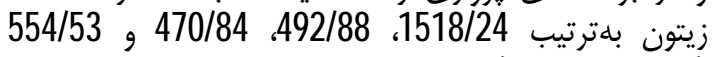
(نانومول در دقيقه) بود. درحالى كه در تحقيق ما فعاليت آنزيم

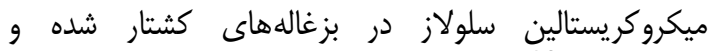

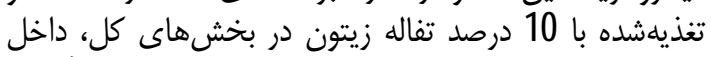

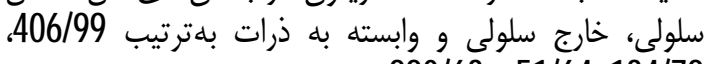

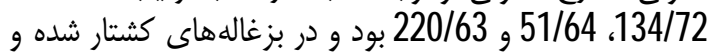

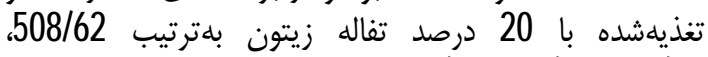
264/62, 78/29،165/71

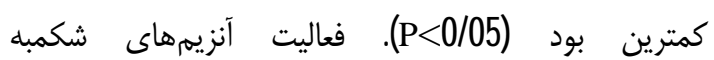

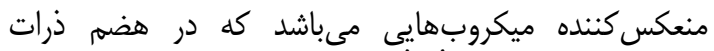

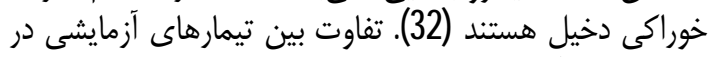

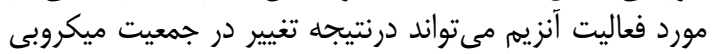

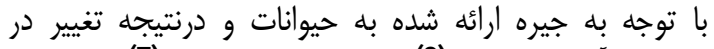

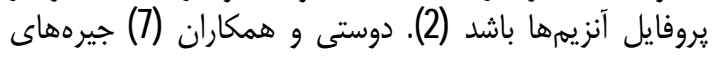

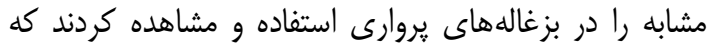

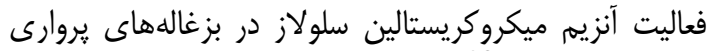

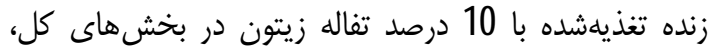
داخل سلولى، خارج سلولى و وابسته به ذرالى ذرات بلهترتيب

جدول2 - اجزاء و تركيب شيميايى جيرههاى آزمايشى مورد استفاده در بزغاله هاى بروارى (درصد از ماده خشى جيره) Table 2. Ingredients and chemical composition of the experimental diets used for fattening goat kids (\% of diet dry matter)

\begin{tabular}{|c|c|c|c|}
\hline \multicolumn{3}{|c|}{ جيرههاى أزمايشى (تفاله زيتون) } & \multirow[b]{2}{*}{ تركيب مواد خوراكى } \\
\hline 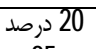 & ل IC درصد & صفر درصد & \\
\hline Z & 2 & 2 & علوفه يونجه \\
\hline $3 \mathrm{C}$ & $4 C$ & $5 C$ & دانه جو \\
\hline 2 & $1 C$ & $\mathrm{C}$ & تفاله زيتون \\
\hline $5 / 6$ & $5 / 90$ & $7 / 45$ & دانه ذرت \\
\hline $1 / 19 E$ & $10 / 66$ & $\mathrm{C} / 0 \mathrm{E}$ & سبوس كندم \\
\hline$\epsilon$ & $\epsilon$ & $\epsilon$ & كنجاله سويا \\
\hline$C / 5 \mathrm{C}$ & $C / 5 C$ & $C / 5 C$ & جوششيرين \\
\hline $\mathrm{C} / 5 \mathrm{C}$ & $C / 5 C$ & $\mathrm{C} / 5 \mathrm{C}$ & نمكى \\
\hline$C / 5 C$ & $C / 5 C$ & $C / 5 C$ & مكمل ويتامينى و معدنى \\
\hline$]$ & 1 & 1 & كربنات كلسيم \\
\hline \multicolumn{4}{|c|}{ تركيب شيميايى (بر اساس (2E) (198E NRC) } \\
\hline 9X/OE & $g / 3 C$ & $8 C / 14$ & ماده خشك \\
\hline $2 / 6$ & $2 / 6$ & $2 / 6$ & انرزى قابل متابوليسم (محاكالرى در كيلوَّرم ماده خشك) \\
\hline 14 & 14 & 14 & 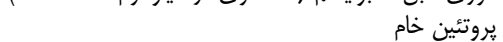 \\
\hline 4/11 & $3 / 3$ & $2 / 54$ & عصاره اترى (Ether Extract) \\
\hline $5 / \pi$ & $5 / 51$ & $5 / 3 x$ & خاكستر (Ash) \\
\hline $4 / 8 C$ & उE/99 & 3C/3C & الياف شوينده خنثى (NDF) \\
\hline $26 / 74$ & $\mathbb{Z} / Z \mathcal{E}$ & $17 / 6 \Subset$ & الياف شوينده اسيدى (ADF) \\
\hline $35 / 32$ & 41/1] & $4 / / 5 \mathrm{C}$ & كربوهيدرات غيراليافى (NFC) \\
\hline C/E & $\mathrm{C} / 8]$ & $\mathrm{C} / 7 \mathrm{C}$ & 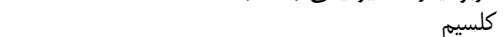 \\
\hline C/34 & $\mathrm{C} / 3 /$ & $C / 3 C$ & فسفر \\
\hline$\pi$ & $\pi$ & $\pi$ & كنسانتره \\
\hline $\mathbf{Z}$ & $\mathbb{Z}^{2}$ & 2 & علوفه \\
\hline
\end{tabular}

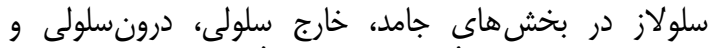

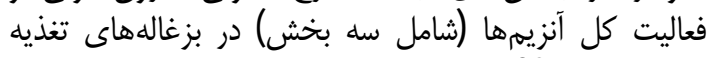

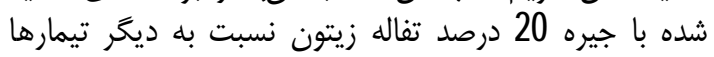

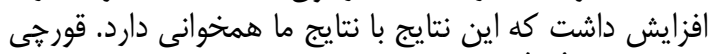

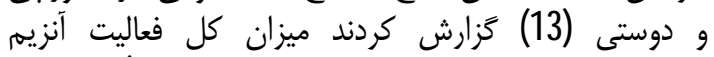

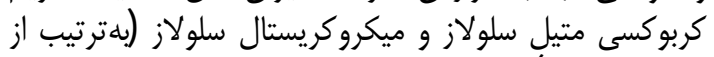

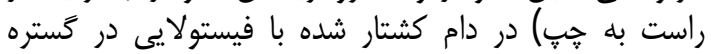

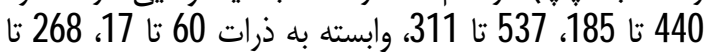

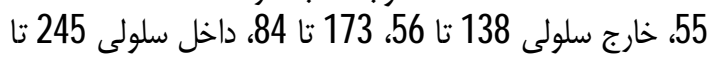

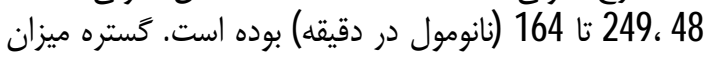

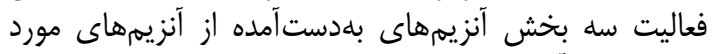

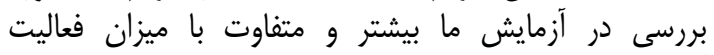

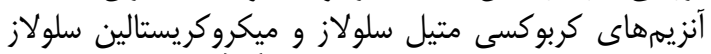
كزارش شده توسط قورجى و دوستى (13) است. اين اين تفاوت

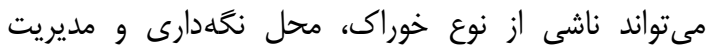

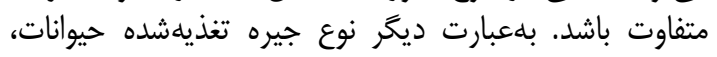

همجنين فعاليت آنزيم كربو كسى متيل سلولاز در يزوهش برو

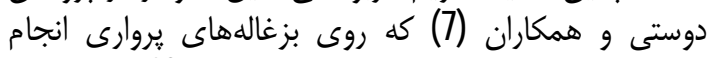

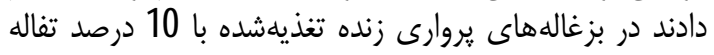

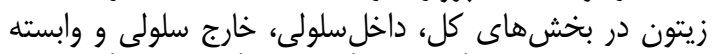

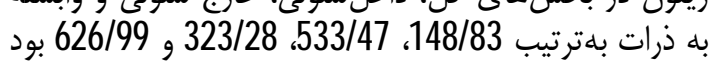

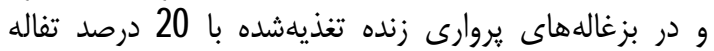

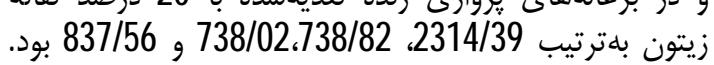

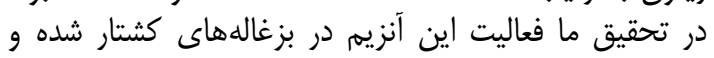

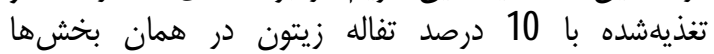

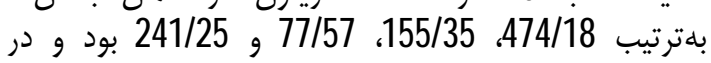

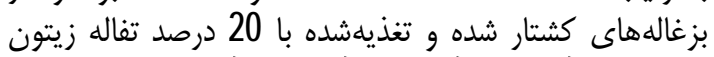

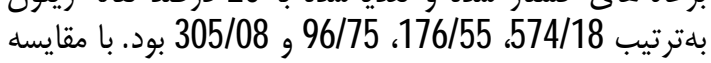

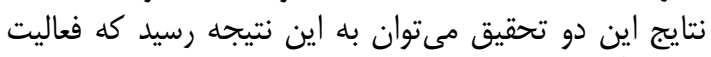

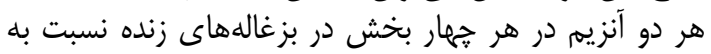

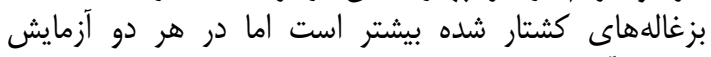
فعاليت آنزيمهاى ميكتروكريستار بيترالين سلولاز و كربو كسى متيل 
بخشهاى مربوطه در آنزيم ميكروكريستالين سلولاز بيشتر

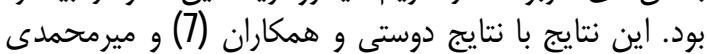

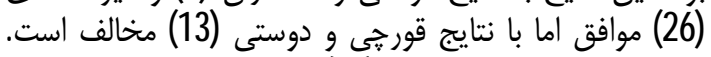

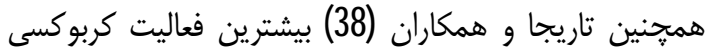

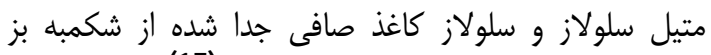

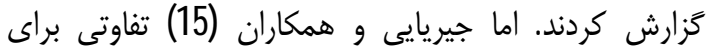

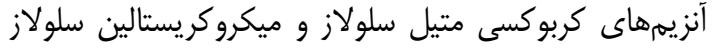

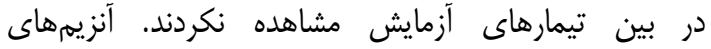

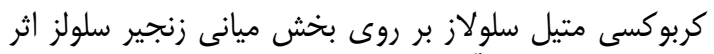

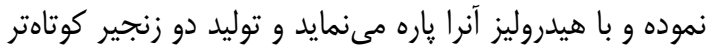

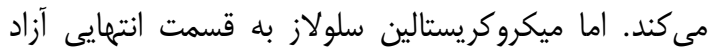

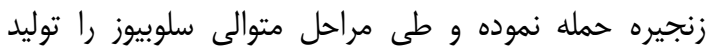

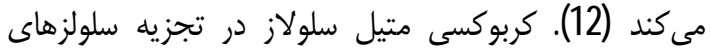

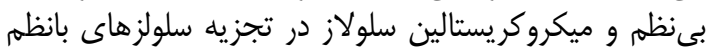

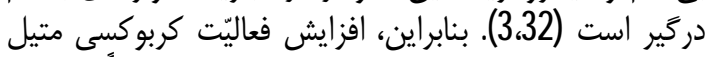

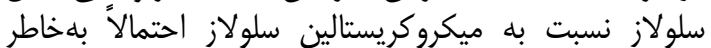

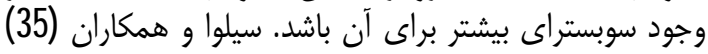
كزارش كردند كه كربوكسى متيل سلولاز بله بلعنوان شان شاخص

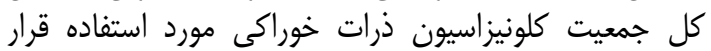

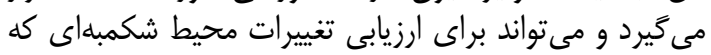

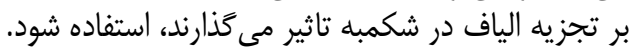

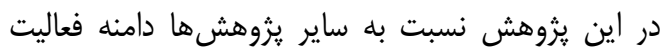

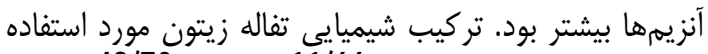

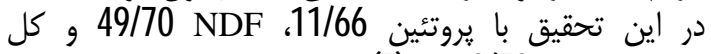

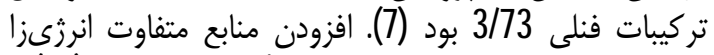

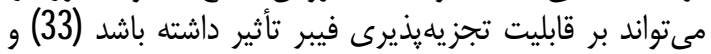

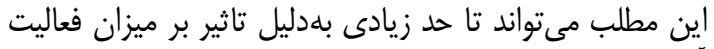

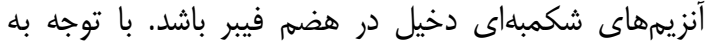

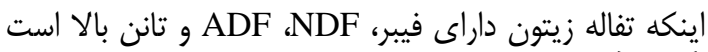

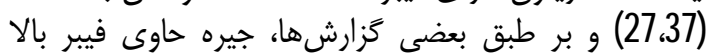

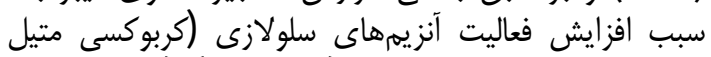

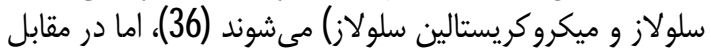

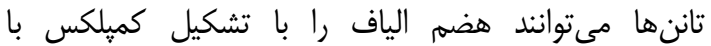

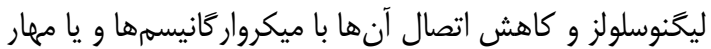

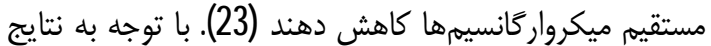

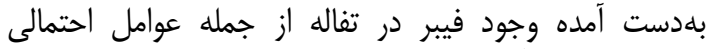

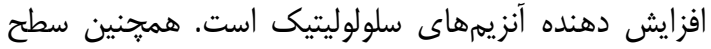

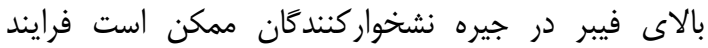

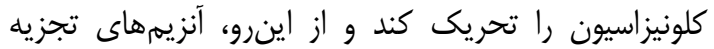

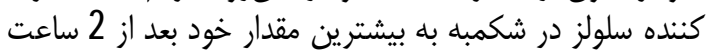

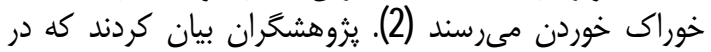

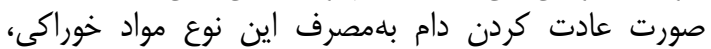

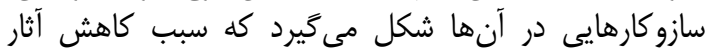

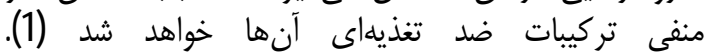

سبب تغيير جمعيت ميكروبى و متعاقب آن تغيير در الكَوى آنس

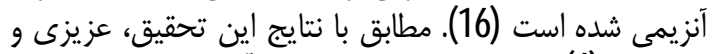

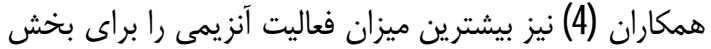

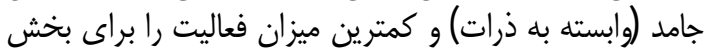

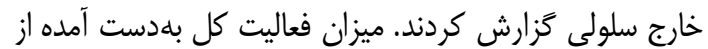

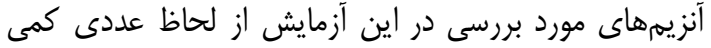

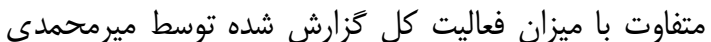

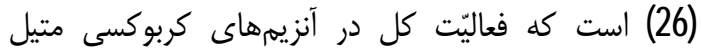

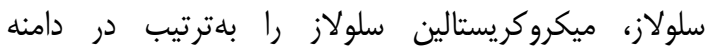

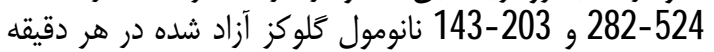

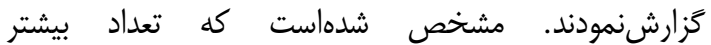

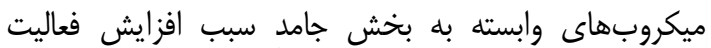

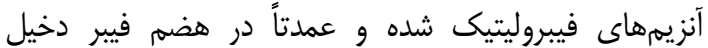

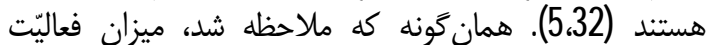

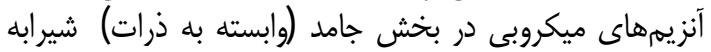

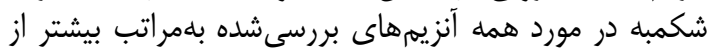

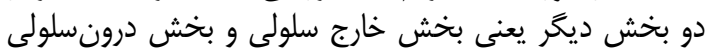

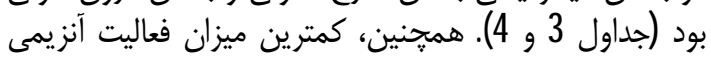

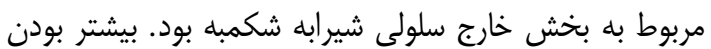

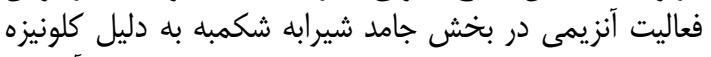

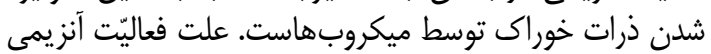

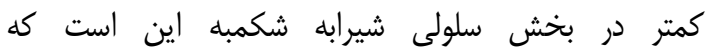

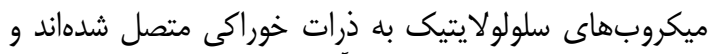

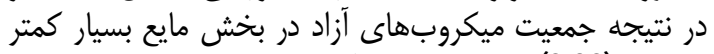

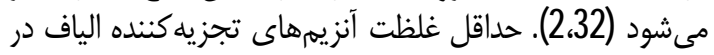

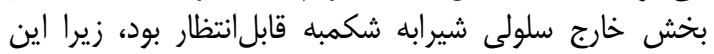

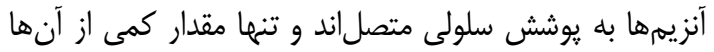

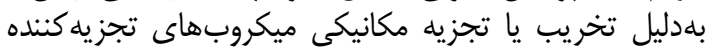

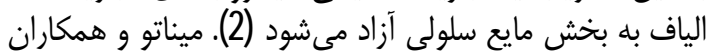

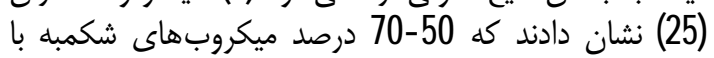

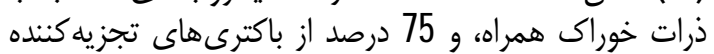

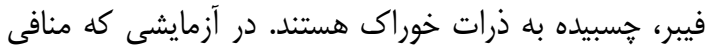

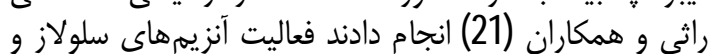

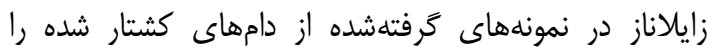

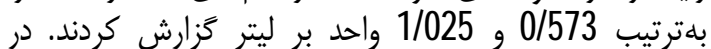

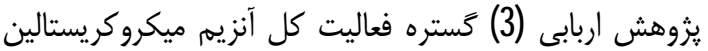

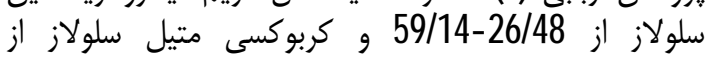

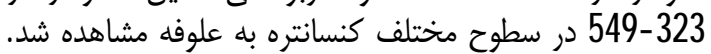

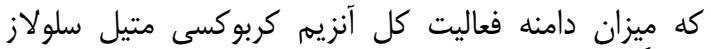

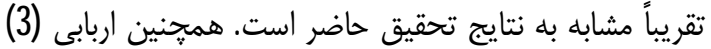

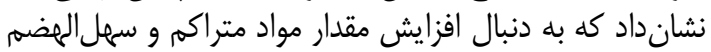

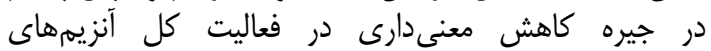

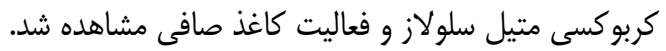

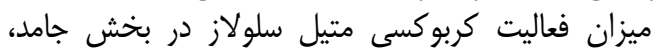
خارج سلولى، درون سلولى و كل شيرابه شكمبه نسبت به به 
جدول3 - تاثير سطوح مختلف تفاله زيتون برفعاليت آنزيم ميكروكريستالين سلولاز (نانومول در دقيقه) در مايع شكمبه بزغالههاى كشتار شده Table 3. Effect of olive cake diets on microcrystalline cellulose enzyme activity (nmol min) in rumen fluid of slaughtered goat kids

\begin{tabular}{|c|c|c|c|c|c|}
\hline \multicolumn{6}{|c|}{ جيرههاى آزمايشى } \\
\hline P-value & SEM & حاوى ح2درصد تفاله زيتون & حاوى 10در صد تفاله زيتون & شاهد & أنزيمها \\
\hline C/000] & $5 / 031$ & $1 \Subset / 7^{\mathrm{a}}$ & $134 T_{2}^{\circ}$ & $11 \varepsilon / 1 \varepsilon^{c}$ & داخل سلولى \\
\hline C/010E & $4 / 85 \varepsilon$ & $7 \varepsilon / \chi^{\mathrm{a}}$ & $51 / 64^{\circ}$ & $35 / 2 k^{c}$ & خارج سلولى \\
\hline C/008] & 1//881 & $264 / \sigma^{a}$ & $220 / 6^{\circ}$ & $16 / / 8 c$ & وابسته به ذرات \\
\hline c/000] & I/31E & $508 / 62^{a}$ & $406 / 99^{\circ}$ & $321 / 2^{c}$ & كل فعاليت \\
\hline
\end{tabular}

جدول4 - تاثير سطوح مختلف تفاله زيتون برفعاليت آنزيم كربوكسى متيل سلولاز (نانومول در دقيقه) در مايع شكمبه بزغالههاى كشتار شده Table 4. Effect of olive cake diets on carboxymethyl cellulose enzyme activity (nmol min) in rumen fluid of slaughtered goat kids

\begin{tabular}{|c|c|c|c|c|c|}
\hline \multicolumn{6}{|c|}{ جيرههاى آزمايشى } \\
\hline P-value & SEM & حاوى X2حر صد تفاله زيتون & حاوى CIدرصد تفاله زيتون & شاهد & أنزيمها \\
\hline (/0001 & $5 / 8 / \varepsilon$ & $17 / 6 / 5^{\mathrm{a}}$ & $15=/ 35^{0}$ & $11 \mathrm{C} / \mathrm{SE}^{\mathrm{c}}$ & داخل سلولى \\
\hline $\mathrm{c} / 000 \mathrm{I}$ & $5 / 091$ & $96 / T^{\mathrm{a}}$ & $7 / 5^{\circ}$ & $4 / / 4 c^{\circ}$ & خارج سلولى \\
\hline c/003 & c/98E & $30 \mathrm{E} / \mathrm{CE}^{\mathrm{a}}$ & $241 / 2^{0}$ & $27 / 2 c^{\circ}$ & وابسته به ذرات \\
\hline (/00) & $1 / 72$ & $5 / 4 / 1 \varepsilon^{\mathrm{a}}$ & $47 / 1 \mathcal{E}^{0}$ & $386 / 21^{\circ}$ & كل فعاليت \\
\hline
\end{tabular}

كاهش جمعيت يروتوزوآيي در شكمبه مىباشد و روغن موجود

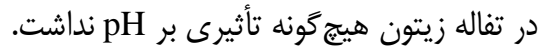

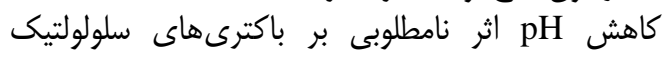

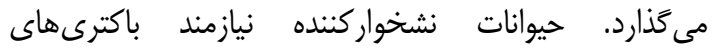

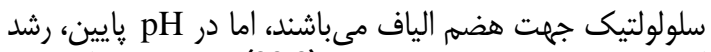
آنها بر سلوبيوز محدود ملىشود (299). اما در اين آزمايش مايش

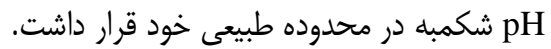

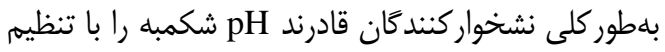

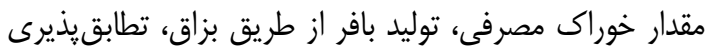

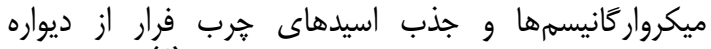

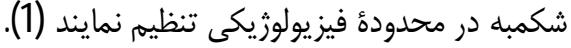

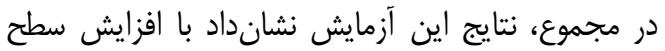

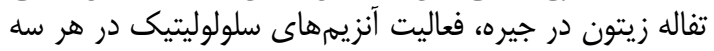

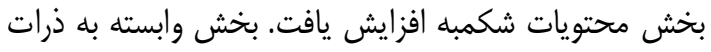

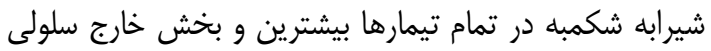

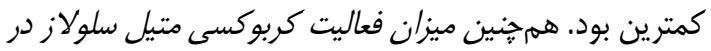

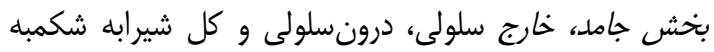

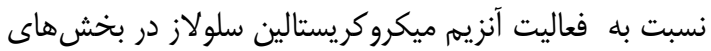
مربوطه بيشتر بود. تثكر و قدردانى

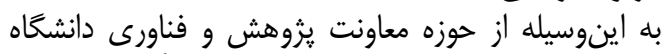

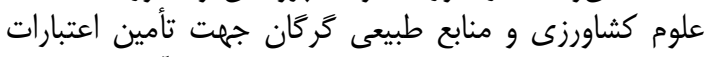

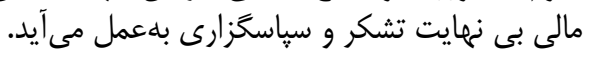

مايع شكمبه pH

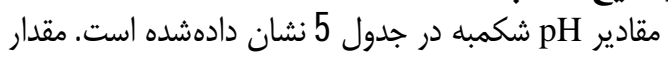

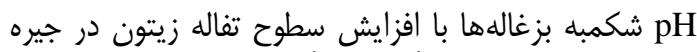

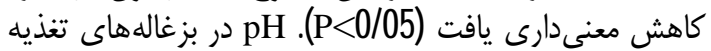

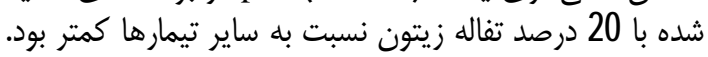

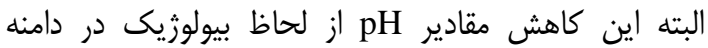

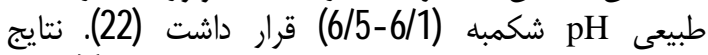

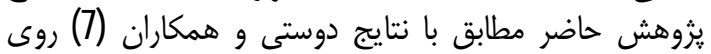

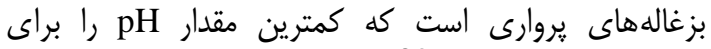

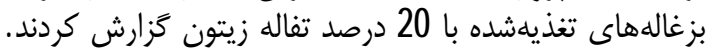

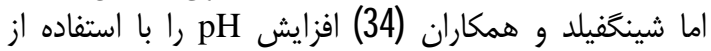

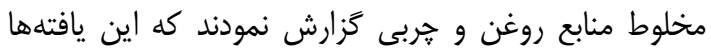

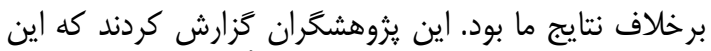
افزايش در pH ممكن است بلهعلت تأثير روغن براف بر بر كاهش

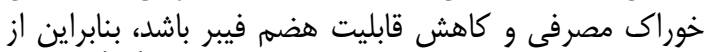

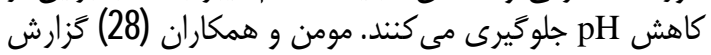
كردند كه جيره شاهد نسبت به جيه جيره

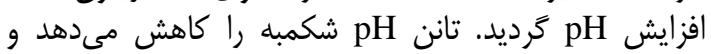

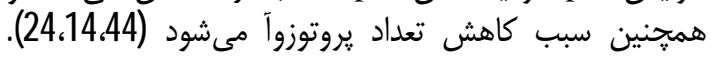

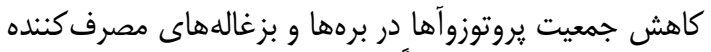

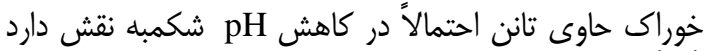

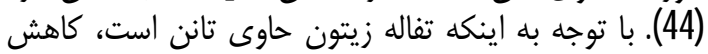
pH

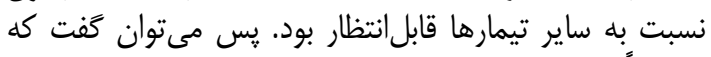

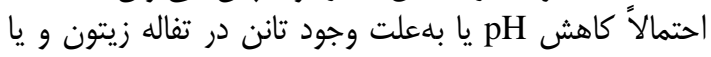


. Acamovic, T. and C.S. Stewart. 2000. Plant phenolic compounds and gastrointestinal micro-organism. In: Brooker, J.D. (Ed.), Tannins in Livestock and Human Nutrition. In: Proceedings of an International workshop held in Adelaide, Australia, 31 May-2 June 1999. Australia Center for International Agricultural Research (ACIAR), Canberra, Australia, 92: 127-129

2. Agarwal, N., I. Agarwal, D.N. Kamra and L.C. Chaudhary. 2000. Diurnal variations in the activities of hydrolytic enzymes in different fractions of rumen contents of murrah buffalo. Journal of Applied Animal Research, 18: 73-80.

3. Arbabi, S. 2013. Effect of forage ratios on bean-based concentrates on fermentation, cellulase enzyme activity and ruminal bacterial population of sheep. Ph.D. Thesis, Gorgan University, Gorgan, Iran. $241 \mathrm{pp}$ (In Persian).

4. Azizi Sotorkhoft, A., A. Safifi, D. Mirmohamadi, J. Rezaei, A. Kiani and H. Fazaeli. 2014. Effect of energy source on some hydrolytic enzymes activities in different fractions of rumen liquor and $\mathrm{N}$ retention in sheep fed diet containing heat-processed broiler litter. Journal of Research in Ruminants, 2: 1-16 (In Persian).

5. Cheng, K.J. and T.A. McAllister. 1997. Comport mentation in the rumen. In The rumen microbial ecosystem. In: Hobson, P.N and C.S. Stewart (eds), Chapman and Hall, London, 492-522.

6. Doi, R.H., A. Kosugi, K. Murashima, Y. Tamaru and S.O. Han. 2003. Cellulosomes from Mesophilic Bacteria. Journal of Bacteriology, 185(20): 5907-5914.

7. Dousti, F., T. Ghoorchi, B. Dastar and A. Azarfar. 2017. Effect of olive cake on performance, rumen characteristics, fibrolytic enzyme and microbial protein synthesis in Najdi goat kids. Animal production research, 6(1): 13-25 (In Persian).

8. Ehsani, M., M.M. Sharifi Hosseini, H. Sadeghi Panah, O. Dayani and M. Asadi Foozi. 2017. The effect of slow-release mineral supplements and eCG injection on twining, birth weight and weaning weigh to fluffy raeini goats. Research on Animal Production, 8(15): 76-83.

9. Enemark, J.M.D. 2008. The monitoring, prevention and treatment of sub-acute ruminal acidosis. Veterinary Journal, 176: 32-43.

10. Eryavuz, A. and B.A. Dehority. 2004. Effect of Yucca schidigera extract on the concentration of rumen microorganisms in sheep. Animal Feed Science and Technology, 117: 215-222.

11. Festenstein, G.N. 1957. Cellulolytic enzymes from sheep-rumen liquor microorganisms. Biochemical Journal, 69(4): 562-567.

12. Ghoorchi, T., B. Ghorbani. 2011. Ruminal microbiology. Publication Gorgan University of Agricultural Sciences and Natural Resources, $169 \mathrm{pp}$.

13. Ghoorchi, T., F. Dousti. 2015. Study of cellulase enzymes activities in rumen fluid of slaughtered fattening lambs. Research report, Gorgan University, Gorgan, Iran, 33 pp (In Persian).

14. Hervás, G., F. PilarFrutos, R. JavierGiráldez Ángel, C. Mantecón Maria and P. AlvarezDel. 2003. Effect of different doses of quebracho tannins extract on rumen fermentation in ewes. Animal Feed Science and Technology, 109: 65-78.

15. Jiriaei, F., M. Kazemi-Bonchari, M.H. Moradi and D. Mirmohamadi. Effect of starch source in diets contained corn steep liquor on performance, blood metabolites, and ruminal enzymes activities of fattening lambs. Research in ruminants, 5(1): 151-168 (In Persian).

16. Kamra, D.N., N. Agarwal and T.A. McAllister. 2010. Screening for compounds enhancing fiber degradation. In: Vercoe, P. E., H.P.S. Makkar, A.C. Schlink (eds.), In vitro Screening of Plant Resources for Extra-nutritional Attributes in Ruminants: Nuclear and Related Methodologies. IAEA, Dordrecht, 85-107.

17. Karami, M. 2018. Effect of diets with different levels of metabolizable energy on physical and chemical carcass chatacteristic of male kids. Research On Animal Production, 9(22): 83-91.

18. Kitts, W.D. and L.A. Underkofler. 1954. Digestion by rumen microorganisms. Hydrolytic products of cellulose and the cellulolytie enzymes. Agricultural Food Chemistry, 2: 639-645.

19. Krause, M.K. and G.R. Otzel. 2006. Understanding and preventing subacute ruminal acidosis in dairy herds: A review. Animal Feed Science and Technology, 126: 215-236.

20. Makkar, H.P.S., A.O. Aderibigbe and K. Becker. 1998. Comparative evaluation of non-toxic and toxic varieties of Jatropha curcas for chemical composition, digestibility, protein degradability and toxic factors. Food Chemistry, 62: 207-215.

21. Manafi Rasi, H., M. Chamani and S. Afshar. 2014. Comparison of methods for dehiscence, concentration and maintenance of digestive enzymes of plant fibers from rumen content of sheep. Animal Production, 16(2): 167-178 (In Persian).

22. McDonald, P., R.A. Edwards, J.F.D. Greenhalgh, C.A. Morgan, L.A. Sinclair and R.G. Wilkinson. 2011. Animal Nutrition. 7th ed., Longman Group UK, Harlow, UK, 693 pp.

23. McSweeney, C.S., B. Palmer, R. Bunch and D.O. Krause. 2001. Effect of the tropical forage Calliandra on microbial protein synthesis and ecology in the rumen. Journal of Applied Microbiology, 90: $78-88$ 
24. Min, B.R., G.T. Attwood, K. Reilly, W. Sun, J.S. Peters, T.N. Barry and W.C. McNabb. 2002. Lotus corniculatus condensed tannins decrease in vivo populations of proteolytic bacteria and affect nitrogen metabolism in the rumen of sheep. Canadian Journal of Microbiology, 48: 911-921.

25. Minato, H., A. Endo, M. Higuchi, Y. Ootomo and T. Uemura. 1966. Ecological treatise on the rumen fermentation. 1. The fractionation of bacteria attached to the rumen digesta solids. The Journal of General and Applied Microbiology, 12: 39-53.

26. Mirmohamadi, D. 2013. Effect of physical form of feed in broiler chickens without broiler diets on yield of lambs. M.Sc. Thesis, Tarbiat Modares University, Tehran, Iran, 120 pp (In Persian).

27. Molina Alcaide, E., D.R. Yañez Ruiz, A. Moumen and I. Martín García. 2003. Chemical composition and nitrogen availability for goats and sheep of some olive by-products. Small Ruminant Research, 49: 329-336.

28. Moumen, A., D.R. Yanez Ruiz, I. Martı́n-Garcı'a and E. Molina Alcaide. 2007. Fermentation characteristics and microbial growth promoted by diets including two-phase olive cake in continuous fermenters. Journal of Animal Physiology and Animal Nutrition, 92: 9-17.

29. Nagaraja, T.G and E.C. Titgemeyer. 2007. Ruminal acidosis in beef cattle: The current microbiological and nutritional outlook. Journal of Dairy Science, 90: 17-38.

30. NRC. 1985. Nutrient Requirements of Small Ruminants. Natl. Acad. Press, Washington, DC.

31. O'Donovan, L. and J.D. Brooker. 2001. Effect of hydrolysable and condensed tannins on growth, morphology and metabolism of Streptococcus gallolyticus (S. caprinus) and Streptococcus bovis. Microbiol, 147: 1025-1033.

32. Raghuvansi, S.K., R. Prasad, M.K. Tripathi, A.S. Mishra, O.H. Chaturvedi, A.K. Mishra, B.L. Saraswat and R.C. Jakhmola. 2007. Effect of complete feed blocks or grazing and supplementation of lambs on performance, nutrient utilisation, rumen fermentation and rumen microbial enzymes.An international of animal bioscience, 1: 221-226.

33. Russell, J.B., J.D.O. Connor, D.G. Fox., P.J. Van Soset and C.J. Sniffen. 1992. A net carbohydrate and protein system for evaluating cattle diets. Ruminal fermentation. Journal of Animal Science, 70: 351-3561.

34. Shingfield, K.J., S. Ahvenjarvi, V. Toivonen, A. Arola, K.V.V. Nurmela, P. Huhtanen and J.M. Griinari. 2003. Effect of dietary fish oil on biohydrogenation of fatty acids and milk fatty acid content in cows. Journal of Animal Science, 77: 165-179.

35. Silva, A.T., R.J. Wallace and E.R. arskov. 1987. Use of particle-bound microbial activity to predict the rate and extent of fibre degradation in the rumen. British Journal of Nutrition, 57: 407-415.

36. Sirohi, S.k., P.K. Choudhury, S.S. Dagar, A.K. Puniya and D. Singh. 2013. Isolation, characterization and fibre degradation potential of anaerobic rumen fungi from cattle. Annals of Microbiology, 63: 1187-1194.

37. Teimouri Yansari, A., H. Sadeghi, Z. Ansari Pirsarai and H. Mohammad Zadeh. 2007. Ruminal dry matter and nutrient degradability of different olive cake by-products after incubation in the rumen using Nylon bag technique. International Journal of Agricultural and Biological, 9: 439-442.

38. Thareja, A., A.K. Puniya, G. Goel, R. Nakgpal, J.P. Sehgal, P.K. Singh and K. Singh. 2006. In vitro degradation of wheat straw by anaerobic fungi from small ruminants. Archives. Anim. Nutrition, 60(5): 412-417.

39. Vaithiyanatha, S., R. Bhatta, A.S. Mishra, R. Prasad, D.L. Verma and N.P. Singh. 2007. Effect of feeding graded levels of Prosopis cineraria leaves on rumen ciliate protozoa, nitrogen balance and microbial protein supply in lambs and kids. Animal Feed Science and Technology, 133: 177-191.

40. Van Soest, P.J. 1994. Nutritional ecology of the ruminant. 2 ed. Comstock Publ. Assoc., Ithaca, NY.

41. Wang, M., X.G. Zhao, Z.L. Tan, S.X. Tang, C.S. Zhou, Z.H. Sun, X.F. Han, and C.W. Wang. 2010. Effects of Increasing Level of Dietary Rice Straw on Chewing Activity, Ruminal Fermentation and Fibrolytic Enzyme Activity in Growing Goats. Asian- Australasian. Journal of Animal Science, 23(8): 1022-1027.

42. Wang, S.P and W.J. Wang. 2016. Effects of dietary supplementation of Chinese herb medicine mixture on rumen fermentation, nutrient digestion and blood profile in goats. South African Journal of Animal Science, 46: 247-260.

43. Wina, E., S. Muetzel and K. Becker. 2005. The dynamics of major fibrolytic microbes and enzyme activity in the rumen in response to short- and long-term feeding of Sapindus rarak saponins. Journal Applied Microbiology, 100: 114-122.

44. Yanez Ruiz, D.R., A. Moumen, A.I. Martin Garcia and E. Molina Alaide. 2004. Ruminal fermentation and degradation patterns, protozoa population and urinary purine derivatives excretion in goats and wethers fed diets based on two stage olive cake: effect of PEG supply. Journal of Animal Science, 82: 2023-2032. 


\title{
Study of Cellulase Enzymes Activity in Rumen Fluid of Fattening Slaughtered Goat Kids
}

\section{Safoura Shahravan ${ }^{1}$ and Taghi Ghoorchi ${ }^{2}$}

1- Ph.D Student, Department of Animal Nutrition,Gorgan University of Agricultural Sciences\&Natural Resources 2- Professor, Gorgan University of Agricultural Sciences\&Natural Resources

(Corresponding author: Ghoorchit@ yahoo.com)

Received: October 27, $2018 \quad$ Accepted: November 12, 2019

\begin{abstract}
An experiment was conducted to investigate the activity of carboxymethylcellulase and microcrystalline-cellulase (Nonomol/min) ruminal hydrolytic enzymes from different fractions of particulate material, extra cellular or cellular. For this purpose, 15 rumen fluids of slaughtered fatteninig Najdi goat kids at the age of 6 months with an average of $28 \mathrm{~kg}$ were sampled in 3 treatments and 5 replicates. The experimental diets included $0,10,20$ percent olive cake was replaced by barley. The results showed that the highest enzyme activity for both enzymes was in the fractions of particulate material in the goat kids fed diet was 20 percent olive cake $(\mathrm{P}<0.05)$. Also, the highest total activity of microcrystalline cellulase and carboxymethyl cellulase enzymes was observed in the goats fed with $20 \%$ olive cake (nmol / min) compared with other treatments $(\mathrm{P}<0.0001)$. Moreover, carboxymethyl-cellulase in particulate material, cellular, extra celluar and total activity where higher than microcrystallinecellulase. The ruminal $\mathrm{pH}$ of goats decreased significantly with the increase in the olive cake in the diet $(\mathrm{P}<0.0001)$. The lowest $\mathrm{PH}$ in goat kids fed with $20 \%$ olive cake was seen compared to other treatments. Overall, the results of this study showed that with increasing levels of olive cake in the diet, the activity of cellulolytic enzymes increased in all three fraction of the rumen contents. The activity of enzymes in particulate material fraction was the highest in all treatments and the extracellular fractions where lowest $(\mathrm{P}<0.05)$.
\end{abstract}

Keywords: Carboxymethyl-Cellulase, Microcrystalline-Cellulase, Enzymes, Slaughtered Goat Kids, Olive Cake 